\title{
Anti-Cytokeratin CAM5.2 Monoclonal Antibody Reacted with Both Cytokeratin 7 and 8, but not Cytokeratin 18. Comment on: Hepatocellular Carcinoma Metastatic to the Gingiva as a First Manifestation of Hepatocellular Carcinoma. J Maxillofac Oral Surg (JMOSI), Published on Line: 25 February 2011; DOI: 10.1007/s12663-011-0187-z
}

\author{
Chih-Ping Han • Ming-Yung Lee
}

Received: 4 July 2011 / Accepted: 8 July 2011 / Published online: 27 July 2011

(C) Association of Oral and Maxillofacial Surgeons of India 2011

\section{To the Editors}

We read with great interest the contribution by Dr. Terada $\mathrm{T}$ regarding the study, entitled "Hepatocellular carcinoma metastatic to the gingiva as a first manifestation of hepatocellular carcinoma" [1]. The author presented a good case report, and implemented a comprehensive discussion. However, there was a typo in the "Abstract" section that CAM5.2 was misspelled "CAM6.2". Additionally, this article contains a wrong identification in the second paragraph of the "Case Report" section that I believe, may misguide the readers. Dr. Terada $\mathrm{T}$ may have inadvertently annotated that "CAM5.2 reacted with both cytokeratin 8 and 18".

We would like to explain that the anti-cytokeratin CAM 5.2, clone CAM 5.2, is marketed by Becton-Dickinson Biosciences (Franklin Lakes, NJ). This clone primarily recognizes both human cytokeratin proteins 7 and 8 , whereas it is lesser specific to cytokeratin 7 . Both peptides

\section{C.-P. Han $(\bowtie)$}

Department of Pathology, Chung-Shan Medical University

Hospital and School of Medicine,

Chung-Shan Medical University, Taichung, Taiwan

e-mail: hanhaly@gmail.com

C.-P. Han

Department of Obstetrics and Gynecology, Chung-Shan Medical

University Hospital, Taichung, Taiwan

C.-P. Han

Clinical Trial Center, Chung-Shan Medical University Hospital, Taichung, Taiwan

M.-Y. Lee

Department of Statistics and Informatics Science,

Providence University, Taichung, Taiwan are found in most epithelial cells, with the exception of stratified squamous epithelium [2].

As a result, it is clarified that anti-cytokeratin CAM5.2 monoclonal antibody is a marker for cytokeratin 7 and 8 but not for cytokeratin 18. This letter can help us elucidate the critical points that CK8/18 is not equivalent to CAM5.2; as well as anticytokeratin CAM5.2 should not be mistaken for monoclonal antibody CK8/18 [2, 3]. However, this finding does not detract from the study results and impressions that hepatocyte paraffin-1 (HepPar-1) (OCH1E5, Dako) is a relatively specific marker of hepatocytes and hepatocellular carcinoma. Hepatocellular carcinoma can manifest as small gingival tumors, and careful hematoxylin-eosin stain and immunoreactive HepPar-1 are useful in the diagnosis of hepatocellular carcinoma in extrahepatic metastatic sites [1].

Conflict of Interests None.

\section{References}

1. Terada $T$ (2011) Hepatocellular carcinoma metastatic to the gingiva as a first manifestation of hepatocellular carcinoma. J Maxillofac Oral Surg (JMOSI). doi: 10.1007/s12663-011-0187-z

2. BD Biosciences anti-cytokeratin (CAM 5.2) reagent for in vitro diagnostic use: 50 tests per vial—catalog no. 349205 . Data sheet, Available at: http://www.bdbiosciences.com/external_files/is/doc/ tds/Package_Inserts_IVD/live/web_enabled/23-3190-02\%20PI_ Anti-Cytokeratin_IVD_EN.pdf and http://www.bdbiosciences.com/ external_files/is/doc/tds/Datasheets_RUO/live/web_enabled/23-133609-347653-347204-349205-DS-ruo.pdf. Accessed 03 July 2011

3. Leica Microsystems. Novocastra ${ }^{\mathrm{TM}}$ Ready-to-use mouse monoclonal antibody cytokeratin (8/18)—product code: RTU-5D3. Available at: http://www.leica-microsystems.com/fileadmin/img_uploads/novocastra_ reagents/Novocastra_datasheets/5d3-r-7-ce.pdf. Accessed 03 July 2011 